\title{
Gestão do Conhecimento aliada ao crescimento organizacional: perspectivas à prática hospitalar
}

\section{Knowledge Management combined with organizational growth: perspectives on hospital practice}

\author{
Rodrigo Pereira ${ }^{1}$ \\ ${ }^{1}$ Faculdade NOVOESTE, Campo Grande, MS, Brasil. ORCID: https://orcid.org/0000-0002-7749-8252
}

Autor para correspondência/Mail to: Rodrigo Pereira, rp121121@hotmail.com

Recebido/Submitted: 07 de julho de 2020; Aceito/Approved: 15 de janeiro de 2021

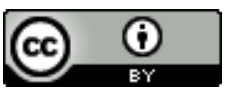

Copyright (C) 2021 Pereira. Todo o conteúdo da Revista (incluindo-se instruções, política editorial e modelos) está sob uma licença Creative Commons Atribuição
4.0 Internacional. Ao serem publicados por esta Revista, os artigos são de livre uso em ambientes educacionais, de pesquisa e não comerciais, com atribuição de autoria obrigatória. Mais informações em http://revistas.ufpr.br/atoz/about/submissions\#copyrightNotice.

\begin{abstract}
Resumo
Introdução: a Gestão do Conhecimento é perceptível no cenário das organizações como uma metodologia capaz de aprimorar a gestão, dando ênfase à informação e ao conhecimento produzidos pelos diversos instanciamentos das organizações como subsídios estratégicos às tomadas de decisão. A partir dessa concepção, a pesquisa em questão partiu do pressuposto de que a Gestão do Conhecimento pode contribuir à gestão hospitalar, sobretudo no que diz respeito aos processos administrativos. Assim sendo, objetivou-se identificar as contribuições da Gestão do Conhecimento, sob a perspectiva dos fluxos de informação, enquanto metodologia para o aprimoramento da gestão hospitalar, bem como, enfatizar o quanto a informação é imprescindível ao crescimento das organizações. Método: para atingir o objetivo acima, buscaram-se, por meio da literatura científica da área, as contribuições da aplicação da Gestão do Conhecimento em ambientes hospitalares. Por meio da literatura da área, pôde-se estabelecer parâmetros oriundos da Gestão do Conhecimento os quais podem sustentar as estratégias de aprimoramento da gestão organizacional hospitalar. A pesquisa se desenvolveu sob a perspectiva exploratória-descritiva, utilizando, sobretudo, das fontes eletrônicas de informação científica disponíveis no cenário nacional, sob o método descritivo para apresentação das análises e resultados. Resultados: a Gestão do Conhecimento, como resultado desta pesquisa, se constitui em significativa ferramenta de aprimoramento dos processos administrativos, podendo contribuir às organizações com maior controle, organização e reuso da informação e do conhecimento produzidos, aperfeiçoando os processos de trabalho, potencializando a gestão e as tomadas de decisão. Conclusão: sugere-se que ferramentas de apoio à sistematização dos fluxos de trabalho sejam estudadas e implementadas nos ambientes de trabalho, vislumbrando sempre maior eficiência, eficácia e efetividade nos fazeres organizacionais hospitalares.
\end{abstract}

Palavras-chave: Gestão do Conhecimento; Gestão da Informação; Fluxos de Informação; Gestão Hospitalar.

\begin{abstract}
Introduction: knowledge Management is perceived in the scenario of organizations as a methodology capable of improving management, emphasizing the information and knowledge produced by the various instances of organizations as strategic subsidies for decision making. From this conception, the research in question started from the assumption that Knowledge Management can contribute to hospital management, especially concerning administrative processes. Therefore, the objective was to identify the contributions of Knowledge Management, from the perspective of information flows, as a methodology for improving hospital management, as well as emphasizing how much information is essential to the growth of organizations. Method: to achieve the above objective, it was sought, through the scientific literature of the area, the contributions of the application of Knowledge Management in hospital environments. Through the literature in the area, it was possible to establish parameters from Knowledge Management that can support strategies for improving hospital organizational management. The research was developed from an exploratory-descriptive perspective, using, above all, the electronic sources of scientific information available in the national scenario, under the descriptive method for presenting the analyzes and results. Results: knowledge Management, as a result of this research, constitutes a significant tool for improving administrative processes and can contribute to organizations with greater control, organization and reuse of the information and knowledge produced, improving work processes, enhancing management and decision making. Conclusion:it is suggested that tools to support the systematization of workflows should be studied and implemented in the work environment, always envisioning greater efficiency, efficacy and effectiveness in hospital organizational activities.
\end{abstract}

Keywords: Knowledge Management; Information management; Information Flow; Hospital Management.

\section{INTRODUÇÃO}

A Gestão da Informação (GI), alinhada à Gestão do Conhecimento (GC), pode melhorar os processos organizacionais das instituições e, conforme defendido por Alves (2017), é essencial para que as organizações sobrevivam no mercado. Neste artigo, apresenta-se a importância de sistematizar os fluxos de trabalho dentro das instituições hospitalares e como será feito o acesso às informações e ao conhecimento gerado por essas instituições.

Beal (2004) afirma que a classificação da informação deve estar baseada em categorias, sejam de informação ou de conhecimento, e possuir sentido para o negócio, permitindo aos usuários várias formas de acesso à informação.

A maneira como os usuários lidam com a informação, ou seja, a manipulam, afeta diretamente a qualidade do fluxo informacional dentro das organizações. A informação, quando transformada em conhecimento, é um ativo da empresa e se torna um recurso para garantir o crescimento das instituições (Beal, 2004).

Por possuir multidisciplinaridade em seus ambientes, as organizações hospitalares se tornam instituições complexas e, por isto, a gestão hospitalar evidencia a necessidade da administração baseada em pressupostos organizacionais, 
capazes de aprimorar os processos gerenciais hospitalares, desvinculando a imagem que antes essas instituições possuíam, que era voltada apenas à filantropia, dando menos ênfase aos processos gerenciais e de negócios.

Implantar a Gestão do Conhecimento, enquanto processo, contribui ao fluxo informacional e de trabalho, permitindo que a instituição identifique melhorias em suas rotinas e assim garanta a qualidade da informação, bem como, institucionalize o conhecimento gerado no âmbito hospitalar, seja ele de caráter operacional, tático e/ou estratégico, potencializando, dessa forma, as tomadas de decisão.

A área administrativa, dentro dos hospitais, acaba sendo a mais silenciosa, entretanto é a que possui todos os recursos estratégicos. É uma área que necessita estar bem estruturada para que os processos fluam pela organização (Moura, Magalhães, \& Chaves, 2001).

Percebe-se que muitas vezes as tecnologias e os Sistemas de Informação (SI) são voltados para a área assistencial, não contribuindo com as ações administrativas, as quais sustentam a missão final dessas instituições.

Hoje, as instituições em geral enfrentam um grande desafio no que diz respeito ao volume de informação, pois geram grandes quantidades de informações, as quais possuem valores significativos. Assim, é necessário identificar e caracterizar os tipos de documentos, bem como o mapeamento dos fluxos informacionais e de trabalho para que se possa identificar falhas e indicar ações que melhor atendam às necessidades da instituição, fazendo o correto uso dessas informações como insumo às estratégias de Gestão do Conhecimento.

Sob essa perspectiva, entende-se que a obtenção de informação implica em processos de trabalho que evidenciem o controle, a organização e, sobretudo, a sistematização dos fluxos informacionais, os quais poderão ser automatizados para que haja ainda mais dinamismo nas tomadas de decisão, contribuindo para a documentação das áreas, tornando o conhecimento explícito e, portanto, gerando insumos ao estabelecimento de práticas de Gestão do Conhecimento.

Muitos processos internos são burocráticos e, em muitas situações, para se obter a resolução de alguma solicitação, há necessidade de percorrer vários setores da instituição, mas não existe controle eficaz da informação produzida. Além disso o conhecimento das áreas se limitam aos colaboradores das referidas áreas, dificultando a localização e acesso às informações para a consolidação das demandas desencadeadas pelas relações de trabalho internas e externas.

Assim sendo, objetivou-se identificar as possíveis contribuições da Gestão do Conhecimento ao aprimoramento dos fluxos de informação hospitalar enquanto processo organizacional.

Para atingir o objetivo acima, houve a necessidade de caracterizar a Gestão do Conhecimento enquanto processo organizacional no âmbito hospitalar e ainda, teve-se que perceber por meio da literatura cientifica da área, as contribuições da aplicação da Gestão do Conhecimento em ambientes hospitalares e, por fim, após alcançados os objetivos foi possível estabelecer parâmetros oriundos da Gestão do Conhecimento como estratégia de aprimoramento da gestão organizacional hospitalar.

A pesquisa se estabeleceu como bibliográfica, utilizando dos referenciais bibliográficos disponíveis no cenário brasileiro, sobretudo das fontes eletrônicas de informações científicas disponíveis via web, sob a perspectiva do método descritivo, o qual suportou a apresentação das análises da pesquisa.

Pôde-se perceber que a Gestão do Conhecimento se constitui em significativa ferramenta de aprimoramento dos processos administrativos, podendo atribuir às organizações maior controle, organização e reuso da informação e do conhecimento produzidos, aperfeiçoando os processos de trabalho, potencializando a gestão e as tomadas de decisão.

Sugere-se, ainda, que as organizações hospitalares criem áreas específicas ao desenvolvimento de boas práticas em Gestão do Conhecimento, implementando novas ferramentas de apoio à sistematização dos fluxos de trabalho, vislumbrando sempre maior eficiência, eficácia e efetividade nos afazeres organizacionais.

\section{A Gestão do Conhecimento enquanto processo organizacional em âmbito hospitalar}

As alterações evidenciadas nos processos de gestão, dando ênfase ao conhecimento como ativo intangível, impulsionaram as organizações à gestão contínua de seus conhecimentos em função da aprendizagem individual e coletiva de seus colaboradores (Oliveira, Castro, \& Brito, 2019).

Com o desenvolvimento crescente das organizações, por meio da evolução tecnológica e da globalização, tem-se utilizado novos termos na administração das organizações e a Gestão do Conhecimento faz parte dessa nova era.

A disseminação e criação da informação é tão rápida e dinâmica que a maneira como esse conhecimento será gerido é de extrema importância nas organizações. Por essa razão, a Gestão do Conhecimento surge com o intuito de agregar valor à informação e facilitar o fluxo das organizações (Teixeira, Pizolotto, Wegner, Albarello, \& Fréo, 2006). 
Corrobora-se a discussão Farias e Araújo (2017, p. 1895) quando afirmam que "A gestão de hospitais no século XXI é invariavelmente complexa, ainda que certos aspectos dos serviços de saúde sejam mais desafiadores em alguns países, como: regulação, financiamento e tecnologias à disposição. Acrescenta-se à extensa relação de demandas gerenciais [...]".

Para Sabbag (2017, p. 60) "a Gestão do Conhecimento nas organizações é um sistema integrado que visa desenvolver conhecimento e competência coletiva para ampliar o capital intelectual de organizações e a sabedoria das pessoas", se bem orientada à missão organizacional, contribui significativamente ao aprimoramento das "demandas gerenciais" citadas por (Farias \& Araújo, 2017).

Angeloni (2008, p. 137) afirma que "o processo de aprendizado organizacional é uma consequência das lições aprendidas de cada indivíduo, uma vez que se caracteriza pela coletividade e pela captura dos conhecimentos de cada membro que reunidos constituem a base de conhecimento organizacional".

Segundo Angeloni (2008, p. 138),

Aprendizagem organizacional é a capacidade de criar novas ideias multiplicada pela capacidade de generalizá-las por toda a empresa. A aprendizagem organizacional corresponde assim, à forma pela qual as organizações constroem, mantêm, melhoram e organizam o conhecimento e a rotina em torno de suas atividades e culturas, a fim de utilizar as aptidões e habilidades da sua força de trabalho de modo cada vez mais eficiente.

Com a descoberta dos resultados proporcionados pela aplicabilidade da Gestão do Conhecimento, as empresas começaram a ter uma visão mais focada no seu capital intelectual para obter inteligência competitiva. Desta forma passam a rever seus processos, suas estratégias e a cultura da organização.

Abbade (2018) afirma que a Gestão do Conhecimento e a aprendizagem organizacional são concepções imbricadas à postura estratégica organizacional, relacionadas às ações que visam manter as organizações atualizadas frente às exigências dos usuários, sejam eles do cenário público e/ou privado, caracterizados como clientes.

Correlatamente, tem-se a Gestão do Conhecimento como um processo potencializador da qualidade dos serviços prestados, assim como, de produtos concebidos no âmago dos processos colaborativos de socialização e cocriação de conhecimento, em que as organizações se preocupam com o conhecimento tácito e sua contribuição durante o ciclo de negócios, uma vez que disseminar o conhecimento do quadro de colaboradores é primordial em qualquer estratégia das empresas.

Sabbag (2017) afirma que "Quanto mais complexa for uma organização, maior a dificuldade de mapear conhecimento e competências, o que não ocorreria caso existissem grupos coesos e estáveis, repositório para todo conhecimento em ação acessível".

Em ambientes hospitalares, é imprescindível a busca pela constante atualização no setor tecnológico para que haja melhorias na qualidade dos serviços prestados. Assim é preciso tratar as informações de forma correta. É evidente que em instituições de saúde existe grande complexidade nos processos administrativos e assistenciais, e que estes devem estar integrados.

Para Ferreira (2007, p. 15):

As organizações podem, seguindo sua capacidade de criação de conhecimento interno e externo, avaliar as estratégias, principalmente quando ocorre troca de informações com outras organizações através de uma rede. Para tanto, torna-se imprescindível avaliar o novo conhecimento em relação ao seu propósito estratégico e suas estimativas de potencial tecnológico e de mercado. Assim, haverá o reconhecimento das inovações operacionais que provocaram a necessidade de um novo sistema social de informações, criando desta forma, um ciclo de informação e conhecimento de renovação constante.

Assim, acredita-se que a tecnologia tem papel estratégico para gerenciar o conhecimento nas organizações e "ajudar o desenvolvimento do conhecimento coletivo e do aprendizado contínuo, tornando mais fácil para as pessoas na organização compartilharem problemas, perspectivas, ideias e soluções" (Teixeira Filho, 2000, p. 12).

A Gestão do Conhecimento está intimamente ligada ao processo de criação e distribuição da informação, independentemente de ela ser tramitada em vários setores de áreas distintas na instituição.

Com o surgimento e a constante evolução da tecnologia, advém também a necessidade de lidar com a complexidade das tarefas nas instituições. A nova realidade fez exigir maior interação entre as pessoas e grupos de diversas áreas de atuação e esta necessidade, em um cenário de ambiente hospitalar, se torna muito mais fatigante pelo nível de estrutura de funções, visto que todos eles atuam em áreas distintas, mas com um mesmo propósito: atender bem e propiciar que o paciente fique satisfeito com a sua estada no hospital. Passos e Aragão (2006), o uso de tecnologias que têm suporte a processo é capaz de contribuir com diversas áreas do conhecimento. O uso da ferramenta Workflow pode ser buscado pelas instituições hospitalares para atender a necessidade de interação e inter-relacionamento entre os setores, por ser capaz de agregar conhecimento e tecnologia atuais, principalmente porque tem o intuito de desenvolver a capacidade intelectual em sistemas de informações. 
Este tipo de sistema apresenta diversas formas de solucionar os problemas enfrentados pelas organizações para melhorar a qualidade de seus fluxos de trabalho, sobretudo no que diz respeito às barreiras existentes nos processos de transferência de conhecimento.

Alguns entraves têm sido notadamente percebidos pela literatura da área como barreiras ao processo de transferência de conhecimento, podendo ser citados a ausência de definição clara das condições nas quais o conhecimento será adquirido e utilizado; a ausência de experiências relacionadas com o conhecimento adquirido; a resistência do destinatário em receber o conhecimento de fora; a falta de motivação em compartilhar o conhecimento; e, ainda, a deficiente capacidade de absorção e problemas de comunicação entre a fonte e o destinatário (Castro, Nascimento, \& Carvalho, 2018).

Outro ponto importante no uso desse recurso é a disseminação da informação, pois sistemas de workflow são projetados para atuarem em ambientes modernos e com múltiplas tarefas interdependentes. Esta característica atende uma das maiores necessidades de um hospital, que é operacionalizar seus processos, os quais são cada vez mais distribuídos entre áreas administrativas, técnicas e assistenciais e, assim, acabam dificultando a gestão da associação de todos esses processos em conjunto (Araújo, 2000).

O uso do workflow pode, também, auxiliar na coordenação das atividades em grupos, pois auxiliam seus partícipes a contribuírem cada qual com sua designação, que juntos concluem seus processos de trabalho. O reconhecimento da ajuda em relação aos participantes do processo e de se ter um fluxo contínuo das atividades além da interação através dos artefatos compartilhados, incentivam seus usuários a compreenderem a importância da sua parte do processo e a executarem com mais zelo.

Desta forma, a instituição hospitalar terá maior domínio na gestão de suas tarefas primordiais, mesmo que sejam complexas, pois contará com um atenuante do conhecimento gerado pela organização. Há a necessidade de registro dos conhecimentos existentes na organização, pois a informação não é perecível, não perde a utilidade em pouco tempo e pode ser acumulada.

Em uma instituição hospitalar, percebe-se um índice alto de rotatividade de funcionários - acima do padrão de organizações empresariais. Neste contexto, os registros do passado, do histórico e da memória organizacional tornam-se ainda mais relevantes, visto que a perda de memória se tornou um problema real. Considerando que um hospital tem um caráter de emergência em sua atividade fim, não há tempo hábil para se ensinar rotinas diárias e, sem uma ferramenta de apoio como workflow, haverá sempre um retrocesso a cada substituição de quadro, fato este que é um prejuízo imenso para uma instituição que é altamente exigida pelo ônus de prover redução de tempo e qualidade de serviço.

Uma aplicação como workflow permite armazenar conhecimento sobre toda organização, pois facilita que grupos de pessoas executem uma mesma atividade em conjunto e por contemplarem o conhecimento organizacional do ambiente onde este fluxo é processado.

O gerenciamento das atividades de uma empresa está fortemente relacionado com a Gestão do Conhecimento. Nonaka e Takeuchi (1997) pressupõe que, assim como a informação, o conhecimento também é específico ao contexto da situação que se encontra e surge de forma dinâmica conforme a interação entre as pessoas daquela organização.

Como em qualquer outra organização, sobretudo em uma instituição hospitalar, ao incentivar o compartilhamento do conhecimento é possível alcançar o objetivo principal de se aplicar a organização da informação, que é objetivar a redução do tempo de todas as rotinas embutidas no processo, diminuir os custos e, com isso, gerar retorno dos investimentos realizados. Esta redução implica maior satisfação dos pacientes de um hospital, pois permanecerão menos tempo no aguardo de um atendimento. Ainda em se tratando de ganhos para a instituição, esta metodologia incentiva o aprendizado contínuo dos profissionais de saúde para a prestação de serviços com mais excelência, o que resulta em melhora da qualidade do atendimento hospitalar.

Segundo Angeloni (2008), a Gestão do Conhecimento, através da aplicação da tecnologia de workflow, pode oportunizar a reestruturação hospitalar no âmbito de seus processos, proporcionando uma visão mais holística das atividades e rotinas hospitalares, em vez de se ancorar em especialização por funções, permitindo assim que estas atividades sejam executadas dentro de processos de normatização. Desta forma, a unidade hospitalar que adotar estes preceitos poderá vislumbrar uma rotina padronizada de todos os membros que prestam serviços.

\section{MATERIAIS E MÉTODOS}

A pesquisa se caracterizou como pesquisa bibliográfica, sob a perspectiva descritiva, por ser desenvolvida a partir de outros materiais elaborados, se sustentando nas principais fontes de informação científica, inclusive as eletrônicas, a fim de possibilitar o alcance da informação de maneira ampla (Gil, 1994).

Optou-se pelo método descritivo, por meio do qual se realizaram as análises dos artigos elencados para esse fim, por meio de leituras com intuito de obter informações que pudessem determinar os materiais relacionados 
aos objetivos da pesquisa, os quais apresentaram cases sobre a Gestão do Conhecimento em âmbito hospitalar (Salvador, 1986).

As análises se sustentaram sob a abordagem qualitativa, por estabelecerem uma compreensão quanto aos dados apresentados, dando-se ênfase aos elementos que pudessem responder a pergunta norteadora desta pesquisa: poderia a Gestão do Conhecimento contribuir à gestão hospitalar, sobretudo no que diz respeito aos processos administrativos de trabalho?

Assim sendo, deu-se a pesquisa entre os meses de abril e julho de 2019, por meio das fontes eletrônicas de informação: Directory of Open Access Journals (DOAJ); OneFile (GALE); Materials Science \& Engineering Database; Scopus (Elsevier) e SciELO (CrossRef). Utilizou-se das palavras-chave: Gestão do Conhecimento, Gestão da Informação, Fluxos de Informação e Gestão Hospitalar, em língua portuguesa e inglesa.

A partir das pesquisas realizadas, chegou-se aos seguintes artigos que denotaram cases sobre a Gestão do Conhecimento nos ambientes hospitalares, em especial, no Brasil: Gestão do Conhecimento: as iniciativas do hospital alemão Oswaldo Cruz; O desenvolvimento de uma prática de Gestão do Conhecimento em um Hospital Geral de Belo Horizonte; Gestão do Conhecimento: Estudo de Caso de um Hospital Universitário e de Ensino; Percepção de cultura organizacional e de Gestão do Conhecimento em hospitais com diferentes modelos de gestão; Diagnóstico das práticas de Gestão do Conhecimento no setor hospitalar, os quais sustentam as análises apresentadas no próximo tópico.

\section{Gestão do Conhecimento aplicada à gestão hospitalar: resultados das análises de cases}

Com o acesso rápido a que todos têm à informação, e com os usuários da saúde cada vez mais tendo ciência de seus direitos, faz-se necessário, cada vez mais, utilizar ferramentas e técnicas que antes eram empregadas apenas no setor industrial e que hoje têm de ser adaptadas para todos os tipos de organizações, inclusive para a área de saúde. Com o grande volume de dados, e buscando preservar o conhecimento, o desenvolvimento e a comunicação nas organizações se tornaram tarefas desafiadoras. A Gestão do Conhecimento pode oportunizar ao profissional da saúde respostas rápidas e assertivas para suas tomadas de decisão, dinamizando os processos de trabalho, bem como, atribuir aos seus afazeres maior grau de assertividade, conformidade e presteza.

A valorização do conhecimento, seja ele implícito e/ou explícito, à medida que faz cada vez mais parte das estratégias das instituições hospitalares, poderá impactar positivamente para a concepção da importância das pessoas enquanto capital intelectual nas instituições, desencadeando mudanças no gerenciamento dos trabalhos, valorizando o capital intelectual e humano, o qual está intimamente atrelado à própria Gestão do Conhecimento.

A necessidade de educação continuada e investimento em capital intelectual não apenas mantém seus profissionais atualizados com a tendência de mercado e com as novas tecnologias de informação e comunicação disponíveis, mas também transforma esse capital em produtores e disseminadores de mais conhecimento.

O ramo da saúde passa por diversas mudanças e avanços tecnológicos e, uma vez que o gestor já saiba como gerir seu conhecimento dentro da instituição hospitalar, há a possibilidade que, diante de uma dificuldade, o gestor faça um comparativo dos dados atuais com dados anteriores e/ou de outras instituições, para facilitar ou nortear a tomada de decisão defronte àquele problema.

Através de literaturas e também de casos bem sucedidos de outras instituições hospitalares, pode-se notar que este tipo de organização possui produção própria de conhecimento, em função de suas vivências e devido à alta complexidade deste tipo de administração. Foi por isto que o objeto de estudo deste artigo voltou-se a pesquisar "cases" de sucesso. Inicia-se abaixo, a explanação do primeiro estudo de caso "As iniciativas do Hospital Alemão Oswaldo Cruz" (Pilla \& Enck, 2009). Este hospital é um dos maiores centros hospitalares da América Latina, foi fundado em 26 de setembro de 1897 por imigrantes de língua alemã, está localizado no bairro Paraíso, em São Paulo (SP) e possui acreditação da Joint Commission.

Pode-se notar que a instituição hospitalar trabalha com modelos, técnicas e métodos de gestão sistematizados e pressupõe iniciativas sólidas e bem estruturadas. O estudo também identificou que a organização de estudo depende da área de Tecnologia de Informação (TI), que pode ser conceituada como base para a Gestão do Conhecimento da organização como um todo.

Evidencia-se, ainda, que na instituição os investimentos em educação continuada, que são fatores determinantes para criar e disseminar o conhecimento, são aplicados tempestivamente. Segundo Bezerra e Leite (2003), a educação continuada é primordial à qualidade da assistência à saúde, principalmente para a enfermagem, e é por este motivo que o hospital iniciou seu programa de educação com foco na equipe de enfermagem, e ratificando a importância e a evolução da equipe, estendeu-se a outras áreas do hospital.

Outro investimento realizado em Gestão do Conhecimento foi a criação de um instituto de ciência com foco na educação da área médica, proporcionando aos colaboradores cursos de extensão e atividades de pesquisa.

Outra prática de Gestão do Conhecimento adotada foi a criação de uma área de "Gerência de Desenvolvimento Institucional" que é responsável em desenvolver a instituição, evoluindo-a para a gestão por processos, imple- 
mentação de projetos e sistemas de garantia da qualidade. Além destes métodos, há investimento e incentivo a congressos e grupos de discussão multidisciplinares entre os profissionais do hospital, de forma a manter o sistema gerando mais conhecimento.

Percebe-se, assim, que há uma cultura organizacional voltada à missão e valores da organização, assim como o desenvolvimento de líderes e pessoas, que são a base da Gestão do Conhecimento da instituição. Todas essas práticas fazem com que a instituição hospitalar alemã Oswaldo Cruz tenha uma estrutura formal para a geração do conhecimento explícito e possua iniciativas para conhecimento tácito.

Não bastassem estas práticas, o hospital Oswaldo Cruz ainda instituiu uma área de lazer para os colaboradores, com pilares conceituais para exercerem atividades como curso de línguas, eventos culturais e oficinas de aprendizado.

Desta forma, o hospital incentiva, de maneira descontraída, os colaboradores a se interessarem em participar destas atividades, contribuindo assim para a construção de um macro conhecimento organizacional, disseminando sempre o conhecimento adquirido.

As iniciativas da Gestão do Conhecimento existentes no Hospital Alemão Oswaldo Cruz corroboram para gerir o conhecimento e proporcionar uma excelente gestão hospitalar.

Outro artigo avaliado foi o dos Autores Cassini e Tomasi (2010), que estudou o Hospital Júlia Kubitschek, criado em 3 de outubro de 1977. Trata-se de um Complexo de Hospital Geral, pertencente à Fundação Hospitalar do Estado de Minas Gerais (FHEMIG), uma das maiores gestoras de hospitais públicos do país, e abrange diversas especialidades de serviços hospitalares prestados à comunidade e, por ser uma instituição pública de saúde, está inserida no SUS.

No estudo realizado, foi observado que as práticas da Gestão do Conhecimento relacionadas a processos ainda estavam em fase de elaboração, planejamento ou implantação. No entanto, a instituição proporciona a seus funcionários educação continuada para aperfeiçoamento e atualização dos conhecimentos, além de cursos e treinamentos que são disponibilizados através de levantamento de necessidades da instituição. Houve um grande empenho para busca de melhores resultados com objetivo de organizar e gerenciar o conhecimento.

Notou-se que a instituição tinha como base tecnológica ferramentas como portais, intranets e extranets as quais serviram de apoio à Gestão do Conhecimento. Identificou-se que nesse período os recursos mais utilizados foram recursos humanos e processos, não sendo feitos grandes investimentos na tecnologia da informação.

Apesar de haver uma preocupação em disponibilizar uma infraestrutura física e equipamentos que dessem suporte a TI e programas que atendessem a necessidade de gerenciamento e disseminação da informação, a necessidade de ampliação e maiores investimentos na área de Tecnologia da Informação era evidente. O estudo reconhece que sistemas de informação são essenciais para inovação e qualidades dos serviços, sendo uma grande vantagem competitiva.

Observa-se que HJK busca constante aprimoramento de suas práticas de Gestão do Conhecimento, com a finalidade de gerir a instituição de forma a sempre alcançar os melhores padrões para a instituição.

Os resultados obtidos a partir da prática de Gestão do Conhecimento na instituição foram a preocupação com produção, retenção e disseminação desse conhecimento adquirido. Dessa forma todo conhecimento poderia ser gerenciado de maneira a proporcionar o auxílio da gestão para tomada de decisão e melhoria do serviço da instituição. Faz-se necessário propiciar a identificação do conhecimento, o gerenciamento e a utilização do mesmo no ambiente organizacional, de maneira a alcançar o desenvolvimento de pessoas e processos, para gerar novos conhecimentos, como auxílio à modernização e inovação da instituição.

Avaliou-se também outro estudo de caso referente à Gestão do Conhecimento, que foi realizado em um Hospital Universitário e de Ensino em Natal/RN. Este estudo teve por objetivo avaliar a percepção dos gestores e chefes do Hospital Universitário e de Ensino em relação à Gestão do Conhecimento na instituição. Segundo a autora da dissertação de mestrado, Oliveira (2011), a justificativa para abordar o tema da Gestão do Conhecimento é a importância para que as instituições obtenham competitividade e sobrevivam às exigências atuais.

A pesquisa de avaliação da Gestão do Conhecimento foi realizada neste universo, e foi aplicado um questionário a um grupo de indivíduos de ambos os sexos, composto pelos diretores, gestores e chefes do hospital de ensino. Ao todo foram entrevistados 100 servidores.

A coleta dos dados foi feita a partir de questionários sociodemográficos, com finalidade de obter a medição da Gestão do Conhecimento (GC), validado no livro "Manual de Gestão do Conhecimento, ferramentas e técnicas que criam valor para a empresa" (DGC), das autoras Bukowitz e Williams (2002). O tempo entre a aplicação e o recolhimento do questionário teve duração de um mês. A pesquisadora teve dificuldade em relação à pesquisa, uma vez que muitos entrevistados dificultaram a entrega do questionário no tempo devido, bem como houve muitos que não sabiam onde haviam colocado o envelope com as perguntas. Depois de recolher os questionários os dados foram inseridos no software Excel, e dispostos em planilhas ilustradas por tabelas e gráficos. 
Ainda segundo Oliveira (2011), o modelo de questionário avaliativo escolhido entre tantos foi baseado na cultura organizacional e o mais adequado acabou sendo das autoras Bukowitz e Williams (2002), já que possui sete etapas essenciais do processo de diagnóstico nos níveis "tático, estratégico e operacional".

Em suas considerações finais, a autora revelou que não houve evidências no Hospital Universitário de "ações formais de Gestão do Conhecimento na organização" e que, a partir dos resultados obtidos, a Gestão do Conhecimento está em fase inicial. Assim, o percentual da instituição foi considerado fraco tanto no "processo tático quanto no estratégico", mostrando que existem várias áreas a serem trabalhadas.

A partir do questionário aplicado, foi possível pontuar que os servidores não têm o hábito de fazer documentações e tão pouco compartilham com exatidão as informações básicas de suas tarefas essenciais e com isso esse conhecimento acaba não sendo compartilhado na organização.

Foi apontada também, a importância das organizações examinarem suas bases de conhecimento frequentemente e assim obterem resultados positivos no que diz respeito ao gerenciamento dos problemas em curto, médio e longo prazo.

Em face de pesquisas realizadas sobre Gestão do Conhecimento, concebe-se que a cultura organizacional é considerada como impactante e pertinente ao sucesso da Gestão do Conhecimento em qualquer tipo de organização, sendo assim, também se concatena com a instituição hospitalar.

Não obstante, para que a cultura da organização de fato corrobore com o sucesso, é necessário que a cultura esteja voltada ao conhecimento, pois, caso não esteja, a própria cultura se constituirá em um empecilho para a eficiência da Gestão do Conhecimento.

Diante destas proposições, procuraram-se relatos que comprovassem esta afirmativa e o resultado foi um estudo de investigação realizado por Cruz e Ferreira (2015), em que foram analisados hospitais com diferentes modelos de gestão e o intuito foi identificar se a percepção dos colaboradores com relação à Gestão do Conhecimento é induzida pela sua percepção da cultura daquela organização.

Para tanto, as autoras utilizaram questionários como instrumento de forma que pudessem fazer a medição desta assimilação da Gestão do Conhecimento e esta aferição foi feita através de um questionário desenvolvido por Cardoso (2003) e também do questionário que mede as práticas da GC utilizado por Almeida, Leal, Pinho, e Fagundes (2006).

A amostra adveio de seis instituições hospitalares públicas da cidade de Coimbra - Portugal, que eram condizentes aos modelos Setor Público Administrativo - chamado de SPA e Entidade Pública Empresarial - chamada de EPE. Os resultados do teste indicam diferenças estatisticamente relevantes nos indicadores de percepção de Gestão do Conhecimento, no que concerne à cultura organizacional, em todas as amostras.

Segundo Cruz e Ferreira (2015), na amostragem realizada nos hospitais EPE, o discernimento de Gestão do Conhecimento assume o índice mais elevado no grupo de entrevistados que identificaram a cultura organizacional como sendo basicamente do tipo clã, comparativamente aos que identificaram outro tipo de cultura como preeminente. Em contrapartida, são os colaboradores que identificaram a cultura de hierarquia como preeminente nos hospitais que trabalham, os que apresentam o índice mais baixo, no que se refere à compreensão de Gestão do Conhecimento.

Na amostragem do modelo SPA, o índice mais elevado de constatação de Gestão do Conhecimento se verifica quando a cultura organizacional é identificada como sendo preeminente do tipo clã, essa cultura apresenta características com maior flexibilidade. Nesse tipo de cultura, a melhor forma de obter bons resultados é por meio de equipes de trabalho, nas quais o papel da liderança é facilitar a participação, comprometimento e lealdade.

Em contrapartida, o índice mais baixo de constatação da Gestão do Conhecimento observa-se quando a cultura organizacional é identificada como sendo majoritariamente uma cultura de mercado. A característica principal desse tipo de cultura organizacional é a competitividade entre membros da mesma organização, e o foco principal está na produtividade, resultados e lucro.

Os resultados obtidos no estudo realizado pelas autoras Cruz e Ferreira (2015) indicam diferenças bastante relevantes nos índices de constatação de Gestão do Conhecimento em concepção à cultura organizacional em todas as amostras, ou seja, analogamente ao que ocorre em outros setores, também na área da saúde, a cultura organizacional interfere diretamente, influenciando a Gestão do Conhecimento. Constatou-se também que o tipo de cultura que mais contribuiu para o surgimento da Gestão do Conhecimento nos hospitais públicos. É a cultura de clã, pois ela possui peculiaridades que viabilizam processos organizacionais relativos ao conhecimento.

Segundo Cruz e Ferreira (2015, p. 82) "os resultados emergentes representam um contributo que pode orientar ações organizacionais e apoiar a decisão dos gestores na implementação de projetos de Gestão do Conhecimento nas instituições hospitalares".

Foi identificado outro estudo sobre o uso de Gestão do Conhecimento no Hospital Clipsi, no município de Campina Grande, Estado da Paraíba, em que se avaliou o conjunto das 18 práticas de Gestão de Conhecimento, 
se são aplicadas ou não, e o seu grau de progresso.

Na figura abaixo, são apresentadas as práticas de Gestão do Conhecimento que estão consolidadas no Hospital Clipsi, totalizando 13 práticas que se enquadram em estágio formal, conforme pesquisa realizada. Segundo Queiroz, Melo, Silva, e de Araújo (2009), "observa-se que as práticas de aprendizagem organizacional, benchmarking ${ }^{1}$ e coaching $^{2}$, estão na categoria de práticas consolidadas, o que representa um elevado grau de formalidade". O resultado denota que a organização hospitalar reconhece a importância de se ter aprendizagem organizacional instituída, pois estas ações contribuem para agilidade em solucionar problemas, que é o objetivo de uma instituição hospitalar que preza pela qualidade dos serviços.

\begin{tabular}{|c|c|}
\hline ESTÁGIO & PRÁTICAS \\
\hline $\begin{array}{l}\text { FORMAL } \\
\text { Práticas Formalizadas } \\
\text { em programas ou atividades na } \\
\text { instituição. }\end{array}$ & $\begin{array}{l}\text { Aprendizagem organizacional } \\
\text { Benchmarking } \\
\text { Coaching } \\
\text { Comunidades de Prática } \\
\text { Comunicação Institucional } \\
\text { Gestão de Competências } \\
\text { Gestão de Marcas e Patentes } \\
\text { Gestão de Relacionamentos com clientes } \\
\text { Inteligência Competitiva } \\
\text { Liçōes Aprendidas } \\
\text { Mentoring } \\
\text { Normalização e Padronização } \\
\text { Portal Corporativo }\end{array}$ \\
\hline PRÁTICAS & CONSOLIDADAS \\
\hline
\end{tabular}

Figura 1. Práticas consolidadas.

Fonte: Queiroz et al. (2009, p. 6).

Práticas como benchmarking e comunicação institucional são utilizadas para atingir metas por meio de ferramentas disponibilizadas aos colaboradores e facilmente consultadas. Além disso o hospital instituiu como meio de compartilhar e disseminar o conhecimento as "Comunidades de Práticas", que trocam as lições aprendidas no dia a dia.

A gestão de competências instaura o engajamento da instituição em planejar e desenvolver as competências relativas ao seu negócio. Todas estas práticas consolidadas ainda têm o arrimo de portais colaborativos para catalogar e compartilhar todas estas informações.

A Figura 2 evidencia que algumas práticas existem no hospital supracitado, porém ainda estão em evolução e em crescimento progressivo.

\begin{tabular}{|l|l|}
\hline \multicolumn{1}{|c|}{ ESTÁGIO } & \multicolumn{1}{c|}{ PRÁTICAS } \\
\hline $\begin{array}{l}\text { EM IMPLANTAÇÃo } \\
\begin{array}{l}\text { Existe e está } \\
\text { em fase de implantação. }\end{array}\end{array}$ & Melhores Práticas \\
\multicolumn{1}{c|}{ PRÁTICAS } & Memória Organizacional \\
\hline
\end{tabular}

Figura 2. Práticas em consolidação.

Fonte: Queiroz et al. (2009, p. 6).

A Figura 3 apresenta as práticas que foram consideradas pouco consolidadas e indicam que o hospital ainda não investiu esforço suficiente para instituir tais práticas de Gestão do Conhecimento. Entretanto, identifica-se a preocupação em manter um acervo do que existe de conhecimento no hospital, pois percebem que estas práticas corroboram para o desempenho do profissional.

Ainda dentre as práticas pouco consolidadas, em contrapartida às anteriores, tem-se que a educação corporativa possui ações em expansão, mas que não são formalizadas.

\footnotetext{
${ }^{1}$ Processo de avaliação de organizações em relação as suas similares, por meio do qual pode-se incorporar as melhores práticas (Pereira, 2019).

${ }^{2}$ Processo sistematizado de autoconhecimento e desenvolvimento de pessoas (Pereira, 2019).
} 


\begin{tabular}{|c|c|}
\hline ESTÁGIO & PRÁTICAS \\
\hline $\begin{array}{l}\text { INFORMAL } \\
\text { Existem açōes relacionadas à prática, mas } \\
\text { elas não estão formalizadas na } \\
\text { instituição }\end{array}$ & $\begin{array}{l}\text { Mapeamento de Conhecimentos } \\
\text { Mapeamento de Procedimento. } \\
\text { Educação Corporativa / Universidade } \\
\text { Corporativa. }\end{array}$ \\
\hline PRÁTICAS & POUCO CONSOLIDADAS \\
\hline
\end{tabular}

Figura 3. Práticas pouco consolidadas.

Fonte: Queiroz et al. (2009, p. 6).

Conclui-se, por meio do estudo feito no Hospital Clipsi - demonstrado nas figuras acima - que o Hospital concebe o quão é notável institucionalizar as práticas de Gestão do Conhecimento em sua organização e, isto posto, proporcionar um ambiente propício e auspicioso para a aplicação das práticas, mesmo aquelas que ainda não estão consolidadas, visto que os colaboradores já têm consciência da importância de desempenhá-las e existem ações em andamento, apenas não formais.

\section{CONCLUSÃO}

Embasado no referencial teórico explicitado neste artigo e por meio de observação inerente às pesquisas, tornou-se possível entender que a partir do momento que a Gestão do Conhecimento é constituída dentro da instituição na qualidade de prática organizacional, esta passará a auxiliar e apoiar a aprendizagem enquanto recurso ao aprimoramento de suas práticas organizacionais, substancializando sua eficácia, eficiência e efetividade por meio de seus processos orgânicos, sobretudo no que diz respeito ao contexto hospitalar.

Foi observado também que não há muito estudo aplicado à Gestão do Conhecimento para organizações hospitalares. Como resultado, as instituições estão deficitárias se comparadas às demais organizações empresariais e, por conseguinte, a ausência de práticas dirigidas à Gestão do Conhecimento distancia a gestão hospitalar de processos capazes de potencializar a produção de conhecimento, bem como dinamizar as decisões administrativas, as quais impactam diretamente na execução da missão das organizações hospitalares.

No decurso de elaboração do artigo, presumiu-se que o compartilhamento de soluções por lições aprendidas no cotidiano é uma prática de suma importância às organizações hospitalares, haja vista que se tratam de organizações complexas com muitas áreas envolvidas nos processos de gestão.

Há profícua rotina de trabalho, entrelaçando profissionais multidisciplinares, em diversos ambientes, o que possibilita alta produção de informação, essas, se bem gerenciadas, poderão sustentar boas práticas de gestão do conhecimento.

A aprendizagem organizacional tem por objetivo preparar estas mesmas pessoas para agir alicerçadas nessa combinação de conhecimentos, experiências vividas, cultura e regras internas, visando identificar as informações que serão oportunas como instrumento decisivo para fomentar o sucesso organizacional.

Por fim, percebe-se, a partir dos cases analisados, que a Gestão do Conhecimento, enquanto processo em uma instituição hospitalar, tende a contribuir à gestão hospitalar facilitando os fluxos informacionais, a geração, tratamento, organização, compartilhamento, uso e reuso do conhecimento para os fins decisórios hospitalares.

Um bom gerenciamento do conhecimento auxilia na melhoria dos serviços prestados pelas instituições de saúde, bem como concebe melhor eficiência e efetividade aos processos de gestão.

Com o monitoramento dos fluxos de informação e estratégias de negócios agrega-se valor à estrutura organizacional da instituição hospitalar e, consequentemente, aos pacientes, que paulatinamente desejam excelência no atendimento. 


\section{REFERÊNCIAS}

Abbade, E. B. (2018). Pesquisa científica como fonte de inovação em hospitais altamente reconhecidos no mundo e no brasil. Gestão da Produção, Operações e Sistemas, 13(2), 231-250. doi: 10.15675/gepros.v13i2.1873.

Almeida, D. A. d., Leal, F., Pinho, A. F. d., \& Fagundes, L. D. (2006). Gestão do conhecimento na análise de falhas: mapeamento de falhas através de sistema de informação. Production, 16(1), 171-188. doi: 10.1590/S010365132006000100014.

Alves, A. (2017). A importância da tecnologia da informação nas empresas. Recuperado de https://www.webartigos.com/artigos/a-importancia-da -tecnologia-da-informacao-nas-empresas/95285/.

Angeloni, M. T. (2008). Organizações do conhecimento: infraestrutura, pessoas e tecnologia. São Paulo: Saraiva.

Araújo, R. M. (2000). Ampliando a cultura de processos de software: um enfoque baseado em groupware e workflow. (Tese de doutorado, Universidade Federal do Rio de Janeiro, Rio de Janeiro, RJ, Brasil). Recuperado de https://www.cos.ufrj.br/index.php/pt-BR/publicacoes -pesquisa/details/15/839

Beal, A. (2004). Gestão estratégica da informação: como transformar a informação e a tecnologia da informação em fatores de crescimento e de alto desempenho nas organizações. São Paulo: Atlas.

Bezerra, A. L. Q., \& Leite, M. M. J. (2003). O contexto da educação continuada em enfermagem. São Paulo: Lemar e Martinari.

Bukowitz, W. R., \& Williams, R. L. (2002). Manual de gestão do conhecimento. São Paulo: Bookman.

Cardoso, L. (2003). Gerir conhecimento e gerar competitividade: estudo empírico sobre a gestão do conhecimento e seu impacto no desempenho organizacional. (Tese de doutorado). Universidade de Coimbra, Coimbra, Portugal.

Cassini, M. R., \& Tomasi, A. (2010). O desenvolvimento de uma prática de gestão do conhecimento em um hospital geral de belo horizonte. Revista de Gestão dos Países de Língua Portuguesa, 9(3). Recuperado de http://www.scielo.mec.pt/scielo.php?script=sci _arttext\&pid=S1645-44642010000200007.

Castro, J. M., Nascimento, M. A., \& Carvalho, R. B. (2018). Transferência de conhecimento tecnológico: análise dos fatores indutores e restritivos à aquisição de conhecimento em dois hospitais do norte de angola. Revista de Administração FACES Journal, 17(4). doi: 10.21714/19846975FACES2018V17N4ART5376.

Cruz, S. G., \& Ferreira, M. M. F. (2015). Percepção de cultura organizacional e de gestão do conhecimento em hospitais com diferentes modelos de gestão. Revista de Enfermagem Referência, 4(5), 75-83. doi: 10.12707/RIV14065.

Farias, D. C., \& Araújo, F. O. d. (2017). Gestão hospitalar no brasil: revisão da literatura visando ao aprimoramento das práticas administrativas em hospitais. Ciência ES Saúde Coletiva, 22(6), 895-1904. doi: 10.1590/141381232017226.26432016 .

Ferreira, V. R. B. (2007). A utilização de práticas de gestão do conhecimento em organizações da sociedade civil que trabalham com projetos de inclusão digital: um estudo de caso (Dissertação de mestrado, Universidade Federal de Santa Catarina, Florianópolis, SC, Brasil). Recuperado de https://repositorio.ufsc.br/xmlui/bitstream/handle/ 123456789/90743/245226.pdf?sequence $=1 \&$ isAllowed $=\mathrm{y}$

Gil, A. C. (1994). Métodos e técnicas de pesquisa social. São Paulo: Atlas.

Moura, G. M. S. S. d., Magalhães, A. M. M. d., \& Chaves, E. H. B. (2001). O serviço de enfermagem hospitalar: apresentando esse gigante silencioso. Revista Brasileira de Enfermagem, 54(3), 482-493. Recuperado de https:// www.scielo.br/pdf/reben/v54n3/v54n3a11.pdf.

Nonaka, I. A., \& Takeuchi, H. (1997). Criação do conhecimento na empresa. Rio de Janeiro: Editora Campus.

Oliveira, J. A. d. (2011). Gestão do conhecimento: estudo de caso de um hospital universitário e de ensino. (Dissertação de mestrado, Universidade Potiguar, Natal, RN, Brasil). Recuperado de https://www.unp.br/wp-content/uploads/ 2013/12/dissertacoes-2009-josicleide-oliveira1.pdf

Oliveira, J. A. d., Castro, A. B. C. d., \& Brito, L. M. P. (2019). Gestão do conhecimento: um estudo em um hospital federal universitário do nordeste do brasil. Figshare. Journal Contribution, 10(2). doi: 10.6084/m9.figshare.7678427.v1.

Passos, D. F. O., \& Aragão, F. S. (2006). Solução workflow para o processo de trabalho no mrp i da hospitalar center. In Anais do $26^{\circ}$ encontro nacional de engenharia de produção. Fortaleza, CE: Abepro. Recuperado de http://www.abepro .org.br/biblioteca/ENEGEP2006_TR450313_7458.pdf.

Pereira, R. (2019). Felicidade, realização e automotivação. Campo Grande: NOVOESTE.

Pilla, B. S., \& Enck, M. A. (2009). Gestão do conhecimento: as iniciativas do hospital alemão oswaldo cruz. Revista Diálogo e Interação, 2. Recuperado de https://www.yumpu.com/pt/document/read/12579271/ gestao-do-conhecimento-as-iniciativas-do-hospital-

Queiroz, C., Melo, A., Silva, S., \& de Araújo, A. (2009). Diagnóstico das práticas de gestão do conhecimento no setor hospitalar. Qualitas Revista Eletrônica, 8(1). doi: 10.18391/qualitas.v8i1.637.

Sabbag, P. Y. (2017). Espirais do conhecimento: ativando indivíduos, grupos e organizações. São Paulo: Saraiva.

Salvador, A. D. (1986). Métodos e técnicas de pesquisa bibliográfica. Porto Alegre: Sulina.

Teixeira, E. B., Pizolotto, M. F., Wegner, T. C. S., Albarello, I., \& Fréo, J. (2006). Gestão do conhecimento em organizações hospitalares: um estudo exploratório. In Anais do $26^{\circ}$ encontro nacional de engenharia de produção. Fortaleza, CE: Abepro. Recuperado de http://www.abepro.org.br/ biblioteca/enegep2006_TR530353_7009.pdf.

Teixeira Filho, J. (2000). Gerenciando conhecimento: como a empresa pode usar a memória organizacional e a inteligência competitiva no desenvolvimento de negócios. Rio de Janeiro: SENAC.

Como citar este artigo (APA):

Pereira, R. (2021). Gestão do Conhecimento aliada ao crescimento organizacional: perspectivas à prática hospitalar. AtoZ: novas práticas em informação e conhecimento, 10(1), 103 -112. Recuperado de: http:// dx.doi.org/10.5380/atoz.v10i1.75082 